Georgian Mathematical Journal

Volume 14 (2007), Number 3, 499-518

\title{
ON SOLVABILITY OF THE NEUMANN PROBLEM IN AN ENERGY SPACE FOR A DOMAIN WITH PEAK
}

\author{
VLADIMIR G. MAZ'YA AND SERGEI V. POBORCHI
}

\begin{abstract}
We describe the dual space of the boundary trace space for functions with a finite Dirichlet integral for a domain with a vertex of an isolated cusp at the boundary. This leads to conditions of solvability of the Neumann problem for elliptic equations of second order. In particular, we give an explicit necessary and sufficient condition for $q$ such that the Neumann problem is solvable if the boundary function is in $L_{q}$ over the boundary of a domain with an outer peak.
\end{abstract}

2000 Mathematics Subject Classification: 35J25, 46 E35.

Key words and phrases: Traces of Sobolev functions, irregular domains, Neumann problem.

\section{INTRODUCTION}

Let $\Omega$ be a domain in $\mathbf{R}^{n}$ and let $W_{2}^{1}(\Omega)$ denote the space of functions on $\Omega$ with the finite norm

$$
\|v\|_{W_{2}^{1}(\Omega)}=\left(\int_{\Omega}\left(|v(x)|^{2}+|\nabla v(x)|^{2}\right) d x\right)^{1 / 2} .
$$

By $T W_{2}^{1}(\Omega)$ we mean the space of boundary traces $\left.u\right|_{\partial \Omega}$ of the functions in $W_{2}^{1}(\Omega)$ with the norm

$$
\|v\|_{T W_{2}^{1}(\Omega)}=\inf \left\{\|u\|_{W_{2}^{1}(\Omega)}: u \in W_{2}^{1}(\Omega),\left.u\right|_{\partial \Omega}=v\right\} .
$$

If $\Omega$ belongs to the class $C^{0,1}$ (i.e., $\Omega$ has a compact closure and its boundary is a locally Lipschitz graph), then Gagliardo's theorem [1] says that $T W_{2}^{1}(\Omega)$ coincides with the space $W_{2}^{1 / 2}(\partial \Omega)$ consisting of functions on $S=\partial \Omega$ having the finite norm

where

$$
\|v\|_{W_{2}^{1 / 2}(S)}=\|v\|_{L_{2}(S)}+[v]_{S}
$$

$$
[v]_{S}=\left(\iint_{S \times S}|v(x)-v(\xi)|^{2} \frac{d s_{x} d s_{\xi}}{|x-\xi|^{n}}\right)^{1 / 2},
$$

and $d s_{x}, d s_{\xi}$ are area elements on $S$.

For $\Omega \in C^{0,1}$ and $S \subset \partial \Omega$ we introduce the space $W_{2}^{-1 / 2}(S)$ of linear continuous functionals on $W_{2}^{1 / 2}(S)$ with the norm

$$
\|f\|_{W_{2}^{-1 / 2}(S)}=\sup \left\{|\langle f, v\rangle|:\|v\|_{W_{2}^{1 / 2}(S)} \leq 1\right\} .
$$

ISSN 1072-947X / \$8.00 / (C) Heldermann Verlag www.heldermann.de 
By Sobolev's theorem the restriction operator

$$
\left.W_{2}^{1}(\Omega) \ni v \mapsto v\right|_{\partial \Omega} \in L_{q}(\partial \Omega), \quad q=2(n-1) /(n-2),
$$

is continuous for $\Omega \in C^{0,1}$, hence $L_{q^{\prime}}(\partial \Omega) \subset W_{2}^{-1 / 2}(\partial \Omega)$ with the minimal possible exponent $q^{\prime}=2(n-1) / n$.

Let $\Omega$ be a domain in $\mathbf{R}^{n}$ of the class $C^{0,1}$. Consider the Neumann problem

$$
\begin{gathered}
-\sum_{i, j=1}^{n} \frac{\partial}{\partial x_{i}}\left(a_{i j}(x) \frac{\partial u}{\partial x_{j}}\right)+a(x) u=0, \quad x \in \Omega, \\
\left.\sum_{i, j=1}^{n} a_{i j}(x) \frac{\partial u}{\partial x_{j}} \cos \left(\nu, x_{i}\right)\right|_{\partial \Omega}=f .
\end{gathered}
$$

We assume that $a_{i j}, i, j=1,2, \ldots, n$, and $a$ are functions in $L_{\infty}(\Omega), a_{i j}=a_{j i}$, $a(x) \geq$ const $>0$ a.e. in $\Omega$, and the following ellipticity condition holds

$$
\sum_{i, j=1}^{n} a_{i j}(x) \xi_{i} \xi_{j} \geq c|\xi|^{2} \quad \text { for all } \xi \in \mathbf{R}^{n}, x \in \Omega
$$

where $c=$ const $>0$. Furthermore, $\nu$ in (1.3) denotes the unit outward normal to $\Omega$ at the point $x \in \partial \Omega$. A function $u \in W_{2}^{1}(\Omega)$ is called a solution of problem $(1.2),(1.3)$ if

$$
[u, v]=\int_{\partial \Omega} f(x) v(x) d s_{x},
$$

for all $v \in W_{2}^{1}(\Omega)$, where $d s_{x}$ is an area element in $\partial \Omega$ and

$$
[u, v]=\int_{\Omega}\left(\sum_{i, j=1}^{n} a_{i j}(x) \frac{\partial u}{\partial x_{j}} \frac{\partial v}{\partial x_{i}}+a(x) u v\right) d x .
$$

In case $f \in W_{2}^{-1 / 2}(\partial \Omega)$, the functional on the right-hand side of (1.4) is continuous with respect to $v \in W_{2}^{1}(\Omega)$ so that the above problem is uniquely solvable.

Suppose that $\Omega$ is a bounded domain whose boundary has a finite number of non-Lipschitz points (for example, there is a vertex of an isolated peak at the boundary). Then, generally $T W_{2}^{1}(\Omega) \neq W_{2}^{1 / 2}(\partial \Omega)$ and hence the space $T W_{2}^{1}(\Omega)^{*}$, dual of $T W_{2}^{1}(\Omega)$, does not coincide with $W_{2}^{-1 / 2}(\partial \Omega)$. Nevertheless, we can set problem (1.2), (1.3) for such $\Omega$ and observe that for $f \in T W_{2}^{1}(\Omega)^{*}$ the right-hand side of $(1.4)$ is again a continuous linear functional in $W_{2}^{1}(\Omega)$. Therefore, the above Neumann problem has a unique solution.

Below, in Section 3 we give a description of the space $T W_{2}^{1}(\Omega)^{*}$ for a domain with a vertex of an outer peak at $\partial \Omega$ in terms of the space $W_{2}^{-1 / 2}$ for Lipschitz surfaces and in terms of some function spaces for interval $(0,1)$ of the real axis. Theorem 1 in Section 3 is the mainre sult of the paper. Its proof is based on the explicit characterization of the space $T W_{2}^{1}(\Omega)$ for a domain with an outer peak (see [3], [4], [5, Ch. 7]). The description of the dual space of $T W_{2}^{1}(\Omega)$ leads to some explicit conditions on the boundary data in (1.3) which provide the solvability of problem (1.2), (1.3). For example, necessary and sufficient 
conditions on $f \in L_{q}(\partial \Omega)$ are given for the Neumann problem to be uniquely solvable in a domain with an outer peak. The case of a domain with an inner peak is considered in Section 4. Section 2 contains some auxiliary assertions.

\section{Notation And Some Lemmas}

We now give the definition of a domain with an outer peak.

Let $\Omega$ be a bounded domain in $\mathbf{R}^{n}(n>2)$. Suppose that the point $O$ belongs to $\partial \Omega$ and the surface $\partial \Omega \backslash\{O\}$ is locally a Lipschitz graph. We locate at $O$ the origin of the Cartesian coordinates $x=(y, z), y \in \mathbf{R}^{n-1}, z \in \mathbf{R}^{1}$. Let $\varphi$ be an increasing function in $C^{0,1}([0,1])$ such that $\varphi(0)=0, \varphi^{\prime}(t) \rightarrow 0$ as $t \rightarrow+0$, and let $\omega$ be a bounded simply connected domain in $\mathbf{R}^{n-1}$ of the class $C^{0,1}$.

Definition. The point $O$ is the vertex of a peak directed into the exterior of $\Omega$ if it has a neighborhood $U$ such that

$$
U \cap \Omega=\{x=(y, z): z \in(0,1), y / \varphi(z) \in \omega\} .
$$

For simplicity, we additionally assume that $\varphi^{\prime}(z) \leq 1 / 2$ for almost all $z \in$ $(0,1)$. We also assume that $\bar{\omega} \subset\{y:|y|<1\}$ and $U \cap \partial \Omega=\{O\} \cup \Gamma$ where

$$
\Gamma=\{x=(y, z): z \in(0,1), y / \varphi(z) \in \partial \omega\} .
$$

We now introduce some notation. Below for brevity we write $T(\Omega)$ instead of $T W_{2}^{1}(\Omega)$ and $T(\Omega)^{*}$ instead of $T W_{2}^{1}(\Omega)^{*}$. If $f \in T(\Omega)^{*}$ and $\lambda$ is a Lipschitz continuous function on $\partial \Omega$, we define

$$
\langle\lambda f, v\rangle=\langle f, \lambda v\rangle, \quad v \in T(\Omega) .
$$

Let $v$ be a function defined on $\Gamma$. Then the mean value of $v$ on the section of $\Gamma$ by the hyperplane $z=$ const is

$$
\bar{v}(z)=\frac{1}{|\gamma|} \int_{\gamma} v(\varphi(z) y, z) d \gamma(y), \quad \gamma=\partial \omega
$$

where $|\gamma|$ is the area of $\gamma$.

If $f \in T(\Omega)^{*}$ and the support of $v \in T(\Omega)$ lies in $\Gamma$, we put

$$
\langle\bar{f}, v\rangle=\langle f, \bar{v}\rangle \text {. }
$$

In what follows some special partition of unity for $\partial \Omega$ will be important for us. To construct it, we define a sequence $\left\{z_{k}\right\}$ by

$$
z_{0} \in(0,1), \quad z_{k+1}+\varphi\left(z_{k+1}\right)=z_{k}, \quad k=0,1, \ldots .
$$

Clearly, $\left\{z_{k}\right\}$ is decreasing and also

$$
z_{k} \rightarrow 0, \quad z_{k+1}^{-1} z_{k} \rightarrow 1, \quad \varphi\left(z_{k+1}\right)^{-1} \varphi\left(z_{k}\right) \rightarrow 1 .
$$

The number $z_{0}$ can be chosen so small that for $z<2 z_{0}$

$$
\varphi(z-2 \varphi(z))>3 \varphi(z) / 4 \text { and } \varphi(z)<z / 4
$$


Let $\left\{\mu_{k}\right\}_{k \geq 1}$ be a smooth partition of unity for $\left(0, z_{1}\right]$ subordinated to the covering by intervals $\Delta_{k}=\left(z_{k+1}, z_{k-1}\right)$, i.e., the set of functions $\mu_{k} \in C_{0}^{\infty}\left(\Delta_{k}\right)$ such that

$$
0 \leq \mu_{k} \leq 1, \quad \sum_{k \geq 1} \mu_{k}(z)=1, \quad z \in\left(0, z_{1}\right] .
$$

This partition of unity can be constructed to satisfy

$$
\operatorname{dist}\left(\operatorname{supp} \mu_{k}, \mathbf{R}^{1} \backslash \Delta_{k}\right) \geq \text { const } \cdot \varphi\left(z_{k}\right), \quad\left|\mu_{k}^{\prime}\right| \leq \text { const } \cdot \varphi\left(z_{k}\right)^{-1}
$$

with constants depending only on $\varphi$.

We introduce another set of functions $\left\{\lambda_{k}\right\}_{k \geq 1}$,

$$
0 \leq \lambda_{k} \leq 1, \quad \lambda_{k} \in C_{0}^{\infty}\left(\Delta_{k}\right),\left.\quad \lambda_{k}\right|_{\operatorname{supp} \mu_{k}}=1 .
$$

Then $\lambda_{k} \mu_{k}=\mu_{k}$ for all $k \geq 1$. Next we define $\mu_{0}(z)=0$ for $z<z_{1}$ and $\mu_{0}(z)=1-\mu_{1}(z)$ for $z \geq z_{1}$. It is clear that then $\sum_{k \geq 0} \mu_{k}(z)=1$ for all $z \in(0,1]$. The partition of unity just constructed as well as the set $\left\{\lambda_{k}\right\}$ depend only on $\varphi$. In what follows we suppose that they are fixed.

Let

$$
\Gamma_{k}=\left\{(y, z) \in \Gamma: z \in \Delta_{k}\right\}, \quad \Delta_{k}=\left(z_{k+1}, z_{k-1}\right), \quad k=1,2 \ldots,
$$

and

$$
\Gamma_{0}=\partial \Omega \backslash\left\{x \in \bar{\Gamma}: z \leq z_{1}\right\} .
$$

We note that the above partition of unity for $(0,1]$ induces a partition of unity for $\partial \Omega \backslash\{O\}$, subordinate to the covering $\left\{\Gamma_{k}\right\}_{k \geq 0}$ if we put $\mu_{0}=1$ on $\Gamma_{0} \backslash \Gamma$.

Let $f \in T(\Omega)^{*}$. If $\langle f, v\rangle=0$ for all $v \in T(\Omega)$ with $\left.v\right|_{\Gamma_{k}}=0$, we say that the support of $f$ lies in $\Gamma_{k}$ and write $\operatorname{supp} f \subset \Gamma_{k}$.

Turning to the study of the space $T(\Omega)^{*}$, we first mention some known results concerning $T(\Omega)$. If $\Omega$ has an outer peak, $T(\Omega)$ can be explicitly characterized as follows (see [3], [4], [5, 7.2]): this space consists of the functions on $\partial \Omega$ with the finite norm

$$
\left(\|v\|_{W_{2}^{1 / 2}\left(\Gamma_{0}\right)}^{2}+\int_{\Gamma} v(x)^{2} \varphi(z) d s_{x}+|v|_{\Gamma}^{2}\right)^{1 / 2},
$$

where $\Gamma$ is given by $(2.1)$,

$$
|v|_{\Gamma}=\left(\int_{\{x, \xi \in \Gamma:|\zeta-z|<M(z, \zeta)\}}|v(x)-v(\xi)|^{2} \frac{d s_{x} d s_{\xi}}{|x-\xi|^{n}}\right)^{1 / 2}
$$

$x=(y, z), \xi=(\eta, \zeta), M(z, \zeta)=\max \{\varphi(z), \varphi(\zeta)\}$, and $d s_{x}, d s_{\xi}$ are the area elements in $\Gamma$. Furthermore the norm in $T(\Omega)$ is equivalent to that in (2.5). The equivalence remains valid if $\Gamma_{0}$ in (2.5) is replaced by the surface $\partial \Omega \backslash\{x \in$ $\bar{\Gamma}: z \leq \delta\}, \delta \in(0,1)$, and the integral over $\Gamma$ is omitted.

Positive quantities $a, b$ are called equivalent or comparable (and written as $a \sim b)$ if $c_{1} \leq a / b \leq c_{2}$ for some positive constants $c_{1}, c_{2}$, depending only on $\Omega$. 
Lemma 1. The following relation holds for $v \in L_{2, l o c}(0,1)$

$$
\begin{gathered}
\iint_{\{x, \xi \in \Gamma:|\zeta-z|<M(z, \zeta)\}}|v(z)-v(\zeta)|^{2} \frac{d s_{x} d s_{\xi}}{|x-\xi|^{n}} \\
\sim \iint_{\{z, \zeta \in(0,1):|\zeta-z|<M(z, \zeta)\}}|v(z)-v(\zeta)|^{2} \frac{M(z, \zeta)^{n-2}}{|z-\zeta|^{2}} d z d \zeta
\end{gathered}
$$

and the estimate

$$
|\bar{v}|_{\Gamma} \leq c(\Omega)\|v\|_{T(\Omega)}
$$

is true for $v \in T(\Omega)$.

Proof. The left-hand side of (2.7) is comparable to

$$
\int_{0}^{1} d z \int_{z-\varphi(z)}^{z}|v(z)-v(\zeta)|^{2}(\varphi(z) \varphi(\zeta))^{n-2} d \zeta \iint_{\gamma \times \gamma} \frac{d \gamma(y) d \gamma(\eta)}{|z-\zeta|^{n}+|\varphi(z) y-\varphi(\zeta) \eta|^{n}},
$$

and furthermore we have for $y, \eta \in \gamma$

$$
|z-\zeta|^{n}+|\varphi(z) y-\varphi(\zeta) \eta|^{n} \sim|z-\zeta|^{n}+\varphi(\zeta)^{n}|y-\eta|^{n} .
$$

Hence

$$
\begin{aligned}
& (\varphi(z) \varphi(\zeta))^{n-2} \iint_{\gamma \times \gamma} \frac{d \gamma(y) d \gamma(\eta)}{|z-\zeta|^{n}+|\varphi(z) y-\varphi(\zeta) \eta|^{n}} \\
& \sim \frac{(\varphi(z) \varphi(\zeta))^{n-2}}{|z-\zeta|^{n}} \int_{\gamma} d \gamma(y) \int_{\gamma} \frac{d \gamma(\eta)}{1+\lambda^{n}|y-\eta|^{n}},
\end{aligned}
$$

where $\lambda=\varphi(\zeta)|z-\zeta|^{-1}$. After the change of variable $\eta=y+\lambda^{-1} t$ in the last integral over $\gamma$ for fixed $y \in \gamma$ the expression on the right-hand side of (2.9) takes the form

$$
\frac{\varphi(z)^{n-2}}{|z-\zeta|^{2}} \int_{\gamma} d \gamma(y) \int_{S_{\lambda}} \frac{d S_{\lambda}(t)}{1+|t|^{n}}
$$

Here $S_{\lambda}$ is the surface $\{t: t / \lambda+y \in \gamma\}$, and $d S_{\lambda}(t)$ the element of the $(n-2)$ dimensional area. It remains to note that $\varphi(z) \sim \varphi(\zeta)$ for $|z-\zeta|<M(z, \zeta)$. Therefore $\lambda \geq$ const $>0$, and the last integral is bounded from above and below uniformly in $\lambda$.

We now turn to (2.8). Using Hölder's inequality, one obtains from (2.2) that

$$
|\bar{v}(z)-\bar{v}(\zeta)|^{2} \leq c \int_{\gamma}|v(\varphi(z) y, z)-v(\varphi(\zeta) y, \zeta)|^{2} d \gamma(y)
$$

The last estimate and (2.7) show that it will suffice to establish the inequality

$$
I_{\gamma}(v) \leq c\|v\|_{T(\Omega)},
$$


where

$$
I_{\gamma}(v)^{2}=\int_{0}^{1} \varphi(z)^{n-2} d z \int_{z-\varphi(z)}^{z} \frac{d \zeta}{|z-\zeta|^{2}} \int_{\gamma}|v(\varphi(z) y, z)-v(\varphi(\zeta) y, \zeta)|^{2} d \gamma(y) .
$$

Let $v=\left.u\right|_{\partial \Omega}$ for some $u \in W_{2}^{1}(\Omega)$. We first establish the estimate

$$
I_{\gamma}(v) \leq c\|\nabla u\|_{L_{2}(\Omega \cap U)}
$$

This estimate is known if $\omega=\{y:|y|<1\}$ (see Lemma 7.6/3 in [5] and Lemma 2.4 in [4]). When $\omega$ is starshaped with respect to a ball centered at the origin, the proof of (2.11) almost verbatim repeats the argument for $\omega=\{y$ : $|y|<1\}$. We omit it.

Let $\omega \subset\left\{y \in \mathbf{R}^{n-1}:|y|<1\right\}$ be starshaped with respect to a ball centered at $y_{0} \in \omega, y_{0} \neq 0$. Then the change of variables

$$
x=(y, z) \mapsto x^{\prime}=\left(y^{\prime}, z^{\prime}\right): z^{\prime}=z, y^{\prime}=y-\varphi(z) y_{0},
$$

transforms $\Omega \cap U$ onto

$$
\Omega^{\prime}=\left\{\left(y^{\prime}, z^{\prime}\right): z^{\prime} \in(0,1), y^{\prime} / \varphi\left(z^{\prime}\right) \in \omega-y_{0}\right\}
$$

where $\omega-y_{0}$ is a domain in $\mathbf{R}^{n-1}$ starshaped with respect to a ball centered at the origin. Let $\tilde{u}$ be defined on $\Omega^{\prime}$ by

$$
\Omega^{\prime} \ni\left(y^{\prime}, z^{\prime}\right) \mapsto \tilde{u}\left(y^{\prime}, z^{\prime}\right)=u\left(y^{\prime}+\varphi\left(z^{\prime}\right) y_{0}, z^{\prime}\right) .
$$

Then $\tilde{u} \in W_{2}^{1}\left(\Omega^{\prime}\right)$. Furthermore we have $\left|\nabla_{x^{\prime}} \tilde{u}\right| \sim\left|\nabla_{x} u\right|$ and $d x^{\prime}=d x$. Hence

$$
\|\nabla \tilde{u}\|_{L_{2}\left(\Omega^{\prime}\right)} \sim\|\nabla u\|_{L_{2}(\Omega \cap U)} .
$$

One can also observe that

$$
I_{\gamma}(v)=I_{\gamma-y_{0}}(\tilde{v}), \quad \tilde{v}=\left.\tilde{u}\right|_{\partial \Omega^{\prime}} .
$$

According to what has been said above, we have

$$
I_{\gamma}(v)=I_{\gamma-y_{0}}(\tilde{v}) \leq c\|\nabla \tilde{u}\|_{L_{2}\left(\Omega^{\prime}\right)} \leq c\|\nabla u\|_{L_{2}(\Omega \cap U)} .
$$

Finally, let $\omega$ be the sum of a finite number of domains starshaped with respect to a ball: $\omega=\bigcup_{i=1}^{N} \omega_{i}$. Here $\gamma=\partial \omega \subset \bigcup_{i=1}^{N} \gamma_{i}, \gamma_{i}=\partial \omega_{i}$ and therefore

$$
I_{\gamma}(v)^{2} \leq \sum_{i=1}^{N} I_{\gamma_{i}}(v)^{2} \leq c\|\nabla u\|_{L_{2}(\Omega \cap U)}^{2}
$$

It remains to note that any domain $\omega \in C^{0,1}$ can be represented as the sum of a finite number of domains starshaped with respect to a ball [2]. So estimate (2.11) follows. To obtain (2.10) for $v \in T(\Omega)$, one should extend given $v$ with finite norm $\|v\|_{T(\Omega)}$ inside $\Omega$ in such a way that for the extended function $u$ we have

$$
\|u\|_{W_{2}^{1}(\Omega)} \leq c\|v\|_{T(\Omega)}
$$

(see [4], [5, 7.2]). Then (2.10) follows from (2.11), which concludes the proof of the lemma. 
Lemma 2. Let $v \in T(\Omega), v(x)=0$ for $x \in \Gamma, z>z_{0}$. Then $|v|_{\Gamma}$ is equivalent to the seminorm

$$
\left(\iint_{\{x, \xi \in \Gamma:|\zeta-z|<2 M(z, \zeta)\}}|v(x)-v(\xi)|^{2} \frac{d s_{x} d s_{\xi}}{|x-\xi|^{n}}\right)^{1 / 2}
$$

with the same notation as in (2.6).

Proof. It suffices to establish the estimate

$$
\iint_{S}|v(x)-v(\xi)|^{2} \frac{d s_{x} d s_{\xi}}{|x-\xi|^{n}} \leq c|v|_{\Gamma}^{2}
$$

where the integration on the left is taken over the set

$$
S=\{(x, \xi) \in \Gamma \times \Gamma: \varphi(z)<z-\zeta<2 \varphi(z)\} .
$$

Since $v(x)=0$ for $z>z_{0}$, it follows that the integrand in (2.12) is not zero only if $z-2 \varphi(z)<z_{0}$. A nondecreasing function $z \mapsto z-2 \varphi(z)$ takes the value no less than $z_{0}$ for $z=2 z_{0}$ because of $(2.3)$. Hence $z-2 \varphi(z)<z_{0}$ only for $z<2 z_{0}$. We also observe that $|x-\xi| \sim \varphi(z)$ for $(x, \xi) \in S$. We establish (2.12) by the so-called fictitious integration. Put

$$
\begin{gathered}
x^{\prime}=\left(y^{\prime}, z^{\prime}\right) \in \Gamma: z^{\prime} \in \delta_{1}(z)=(z-\varphi(z), z-3 \varphi(z) / 4), \\
\xi^{\prime}=\left(\eta^{\prime}, \zeta^{\prime}\right) \in \Gamma: \zeta^{\prime} \in \delta_{2}(z)=(z-3 \varphi(z) / 2, z-5 \varphi(z) / 4) .
\end{gathered}
$$

Then

$$
0<z-z^{\prime}<\varphi(z)=M(z, \zeta)
$$

In view of (2.3) we have

$$
0<z^{\prime}-\zeta^{\prime}<3 \varphi(z) / 4<\varphi(z-3 \varphi(z) / 2)<\varphi\left(z^{\prime}\right)=M\left(z^{\prime}, \zeta^{\prime}\right)
$$

and

$$
\left|\zeta^{\prime}-\zeta\right| \leq 3 \varphi(z) / 4<\varphi\left(\zeta^{\prime}\right) \leq M\left(\zeta, \zeta^{\prime}\right)
$$

Integrating the inequality

$$
c|v(x)-v(\xi)|^{2} \leq\left|v(x)-v\left(x^{\prime}\right)\right|^{2}+\left|v\left(x^{\prime}\right)-v\left(\xi^{\prime}\right)\right|^{2}+\left|v\left(\xi^{\prime}\right)-v(\xi)\right|^{2}
$$

with respect to $x^{\prime}, \xi^{\prime}$ and using the fact that any of the quantities

$$
\left|x-x^{\prime}\right|,\left|x^{\prime}-\xi^{\prime}\right|,\left|\xi^{\prime}-\xi\right|
$$

does not exceed $c|x-\xi|$ (which is comparable to $\varphi(z)$ ), we obtain

$$
\begin{aligned}
& c \frac{|v(x)-v(\xi)|^{2}}{|x-\xi|^{n}} \leq \frac{1}{\varphi(z)^{n-1}} \int_{\left\{x^{\prime} \in \Gamma: z^{\prime} \in \delta_{1}(z)\right\}}\left|v\left(x^{\prime}\right)-v(x)\right|^{2} \frac{d s_{x^{\prime}}}{\left|x^{\prime}-x\right|^{n}} \\
& +\frac{1}{\varphi(z)^{2(n-1)}}\left|v \int_{\left\{x^{\prime}, \xi^{\prime} \in \Gamma: z^{\prime} \in \delta_{1}(z), \zeta^{\prime} \in \delta_{2}(z)\right\}}\right| v\left(x^{\prime}\right)-\left.v\left(\xi^{\prime}\right)\right|^{2} \frac{d s_{x^{\prime}} d s_{\xi^{\prime}}}{\left|x^{\prime}-\xi^{\prime}\right|^{n}} \\
& +\frac{1}{\varphi(z)^{n-1}} \int_{\left\{\xi^{\prime} \in \Gamma: \zeta^{\prime} \in \delta_{2}(z)\right\}}\left|v\left(\xi^{\prime}\right)-v(\xi)\right|^{2} \frac{d s_{\xi^{\prime}}}{\left|\xi^{\prime}-\xi\right|^{n}} .
\end{aligned}
$$


In view of (2.13)-(2.15) the integration of the last inequality over $S$ combined with Fubini's theorem gives (2.12). The proof of the lemma is concluded.

Remark 1. It follows from (2.5) that the linear map $T(\Omega) \ni v \mapsto \psi v \in T(\Omega)$ is continuous if $\psi$ is Lipschitz continuous on $\partial \Omega^{1}$.

In what follows we need a version of the "Poincaré inequality" for functions defined on surfaces. Let $\sigma$ be a measurable subset of the boundary of a domain of the class $C^{0,1}$ with positive area $|\sigma|$.

Lemma 3. If $v \in L_{2}(\sigma)$, then

$$
\|v-\tilde{v}\|_{L_{2}(\sigma)}^{2} \leq(\operatorname{diam} \sigma)^{n}|\sigma|^{-1}[v]_{\sigma}^{2},
$$

where $\tilde{v}$ is the mean value of $v$ on $\sigma$ :

$$
\tilde{v}=|\sigma|^{-1} \int_{\sigma} v(x) d s_{x}
$$

and $[\cdot]_{\sigma}$ is the seminorm defined in (1.1).

This assertion easily follows by Hölder's inequality.

\section{Space $T W_{2}^{1}(\Omega)^{*}$ For a Domain with an Outer Peak}

The theorem stated below gives a description of the space $T(\Omega)^{*}$ dual of $T(\Omega)$ for a domain with an outer peak. To state the theorem, we need a new space of functions defined on the interval $(0,1)$ of the real axis. Let $\bar{W}_{2}^{1 / 2}(0,1)$ be the space of functions in $L_{2, l o c}(0,1)$ having the finite norm

$$
\begin{gathered}
\|u\|_{\bar{W}_{2}^{1 / 2}(0,1)}=\left(\int_{0}^{1} u(z)^{2} \varphi(z)^{n-1} d z\right. \\
\left.+\quad \iint_{\{z, \zeta \in(0,1):|\zeta-z|<M(z, \zeta)\}}|u(z)-u(\zeta)|^{2} \frac{M(z, \zeta)^{n-2}}{|z-\zeta|^{2}} d z d \zeta\right)^{1 / 2},
\end{gathered}
$$

where, as before, $M(z, \zeta)=\max \{\varphi(z), \varphi(\zeta)\}$.

Theorem 1. Let $\Omega \subset \mathbf{R}^{n}$ be a domain with an outer peak and $\left\{\mu_{k}\right\}_{k \geq 0}$ the partition of unity for $\partial \Omega \backslash\{O\}$ constructed above.

(i) If $f \in T(\Omega)^{*}$, then $f$ can be represented as the sum of three summands

$$
f=\mu_{0} f+\left(1-\mu_{0}\right) \bar{f}+\left(1-\mu_{0}\right)(f-\bar{f})=f^{(1)}+f^{(2)}+f^{(3)}
$$

\footnotetext{
${ }^{1}$ We arrive at the same conclusion for an arbitrary domain if we use the following fact: a function, which is Lipschitz continuous on $\partial \Omega$, can be extended to a Lipschitz continuous function on $\mathbf{R}^{n}$ with the same Lipschitz constant and the same maximum of the modulus (Stein; see [8], Ch. VI, § 2). Then

$$
\begin{gathered}
\|\psi v\|_{T W_{2}^{1}(\Omega)}=\inf \left\{\|u\|_{W_{2}^{1}(\Omega)}:\left.u\right|_{\partial \Omega}=\psi v\right\} \leq \inf \left\{\|\psi u\|_{W_{2}^{1}(\Omega)}:\left.u\right|_{\partial \Omega}=v\right\} \\
\leq c(\psi) \inf \left\{\|u\|_{W_{2}^{1}(\Omega)}:\left.u\right|_{\partial \Omega}=v\right\}=c(\psi)\|v\|_{T W_{2}^{1}(\Omega)} .
\end{gathered}
$$
}


in $T(\Omega)^{*}$ with the following properties. The support of $f^{(1)}$ lies in $\Gamma_{0}$ and $f^{(1)} \in$ $W_{2}^{-1 / 2}\left(\Gamma_{0}\right)$. The term $f^{(2)}$ defined by

$$
T(\Omega) \ni v \mapsto\left\langle f^{(2)}, v\right\rangle=\left\langle f,\left(1-\mu_{0}\right) \bar{v}\right\rangle,
$$

has the support in $\left\{x \in \Gamma: z \leq z_{0}\right\}$ and belongs to $\bar{W}_{2}^{1 / 2}(0,1)^{*}$ in the sense that

$$
\left|\left\langle f^{(2)}, v\right\rangle\right| \leq \text { const } \cdot\|\bar{v}\|_{\bar{W}_{2}^{1 / 2}(0,1)} .
$$

The support of $f^{(3)}$ lies in $\left\{x \in \Gamma: z \leq z_{0}\right\}$, the decomposition

$$
\left\langle f^{(3)}, v\right\rangle=\sum_{k \geq 1}\left\langle\mu_{k}(f-\bar{f}), v\right\rangle, \quad v \in T(\Omega)
$$

holds and the estimate

$$
\left(\sum_{k \geq 1}\left\|\mu_{k}(f-\bar{f})\right\|_{W_{2}^{-1 / 2}\left(\Gamma_{k}\right)}^{2}\right)^{1 / 2} \leq c\left\|\left(1-\mu_{0}\right)(f-\bar{f})\right\|_{T(\Omega) *}
$$

with a constant depending only on $\Omega$ is valid.

(ii) Let $f_{k} \in W_{2}^{-1 / 2}\left(\Gamma_{k}\right)$ for $k \geq 1$. Suppose that $\operatorname{supp} f_{k} \subset \Gamma_{k},\left\langle f_{k}, v\right\rangle=0$ if $v \in W_{2}^{1 / 2}\left(\Gamma_{k}\right), v(y, z)$ depends only on $z$, and

$$
\sum_{k \geq 1}\left\|\lambda_{k} f_{k}\right\|_{W_{2}^{-1 / 2}\left(\Gamma_{k}\right)}^{2}<\infty
$$

Define

$$
T(\Omega) \ni v \mapsto\left\langle f^{(3)}, v\right\rangle=\sum_{k \geq 1}\left\langle\lambda_{k} f_{k}, v\right\rangle .
$$

Then $f^{(3)}$ belongs to $T(\Omega)^{*}$, its support lies in $\left\{x \in \Gamma: z \leq z_{0}\right\}$ and the estimate

$$
\left\|f^{(3)}\right\|_{T(\Omega)^{*}} \leq c\left(\sum_{k \geq 1}\left\|\lambda_{k} f_{k}\right\|_{W_{2}^{-1 / 2}\left(\Gamma_{k}\right)}^{2}\right)^{1 / 2}
$$

with a constant depending only on $\Omega$ holds. Furthermore, let $h \in W_{2}^{-1 / 2}\left(\Gamma_{0}\right)$ and $g \in \bar{W}_{2}^{1 / 2}(0,1)^{*}$. Define $f^{(1)}=\mu_{0} h$,

$$
\left\langle f^{(2)}, v\right\rangle=\left\langle g,\left(1-\mu_{0}\right) \bar{v}\right\rangle, \quad v \in T(\Omega) .
$$

Then $f^{(1)}, f^{(2)} \in T(\Omega)^{*}$ and moreover $f^{(1)} \in W_{2}^{-1 / 2}\left(\Gamma_{0}\right)$.

Proof. (i) The inclusion $f^{(1)}=\mu_{0} f \in T(\Omega)^{*}$ follows from Remark 1. Next, we have

$$
\left|\left\langle f^{(1)}, v\right\rangle\right|=\left|\left\langle f, \mu_{0} v\right\rangle\right| \leq c\|f\|_{T(\Omega)^{*}}\left\|\mu_{0} v\right\|_{W_{2}^{1 / 2}\left(\Gamma_{0}\right)}
$$

because $\|v\|_{T(\Omega)} \sim\|v\|_{W_{2}^{1 / 2}\left(\Gamma_{0}\right)}$ for the functions supported in $\Gamma_{0}$. Gagliardo's theorem and Remark 1 also imply that

$$
\left\|\mu_{0} v\right\|_{W_{2}^{1 / 2}\left(\Gamma_{0}\right)} \leq c\|v\|_{W_{2}^{1 / 2}\left(\Gamma_{0}\right)}
$$

Thus $f^{(1)} \in W_{2}^{-1 / 2}\left(\Gamma_{0}\right)$. 
Consider the term $f^{(2)}$. Here

$$
\left|\left\langle f^{(2)}, v\right\rangle\right|=\left|\left\langle f,\left(1-\mu_{0}\right) \bar{v}\right\rangle\right|,
$$

where $\bar{v}=\bar{v}(z)$ is the mean value of $\left.v\right|_{\Gamma}$, defined in (1.2). By using the continuity of $f$ and the equivalence $\|v\|_{T(\Omega)} \sim|v|_{\Gamma}$ for $v$ supported in $\left\{x \in \Gamma: z \leq z_{0}\right\}$, we dominate quantity $(3.7)$ by expression $c(f)\left|\left(1-\mu_{0}\right) \bar{v}\right|_{\Gamma}$ (recall that the seminorm $|\cdot|_{\Gamma}$ is defined in (2.6)). An application of Lemma 1 gives the following majorant for the square of quantity (3.7):

$$
c \iint_{S}\left|\left(1-\mu_{0}(z)\right) \bar{v}(z)-\left(1-\mu_{0}(\zeta)\right) \bar{v}(\zeta)\right|^{2} M(z, \zeta)^{n-2} \frac{d z d \zeta}{|z-\zeta|^{2}},
$$

where $S=\{(z, \zeta) \in(0,1) \times(0,1):|z-\zeta|<M(z, \zeta)\}$. We dominate expression (3.8) by the sum

$$
\begin{aligned}
c \iint_{S} \frac{|\bar{v}(z)-\bar{v}(\zeta)|^{2}}{|z-\zeta|^{2}} & M(z, \zeta)^{n-2} d z d \zeta \\
& +c \iint_{S} \frac{\left|\mu_{0}(z)-\mu_{0}(\zeta)\right|^{2}}{|z-\zeta|^{2}} \bar{v}(z)^{2} M(z, \zeta)^{n-2} d z d \zeta .
\end{aligned}
$$

The last term does not exceed

$$
c \int_{0}^{1} \bar{v}(z)^{2} \varphi(z)^{n-1} d z
$$

which is not greater than $c \int_{\Gamma} v(x)^{2} \varphi(z) d s_{x}$, and we arrive at (3.1) with a constant independent of $v$. It remains to observe that the right-hand side of (3.1) does not exceed $c\|v\|_{T(\Omega)}$ in view of Lemma 1 . Hence $f^{(2)} \in T(\Omega)^{*}$.

We now consider $f^{(3)}$. The inclusion $f^{(3)} \in T(\Omega)^{*}$ is a consequence of the inclusions $f^{(1)}, f^{(2)} \in T(\Omega)^{*}$. To check (3.2), we choose an element $v_{k} \in W_{2}^{1 / 2}\left(\Gamma_{k}\right)$, $k=1,2, \ldots$, such that $\left\|v_{k}\right\|_{W_{2}^{1 / 2}\left(\Gamma_{k}\right)} \leq 1$ and

$$
\left\|\mu_{k}(f-\bar{f})\right\|_{W_{2}^{-1 / 2}\left(\Gamma_{k}\right)} \leq 2\left\langle\mu_{k}(f-\bar{f}), v_{k}\right\rangle \text {. }
$$

Put $\alpha_{k}=\left\|\mu_{k}(f-\bar{f})\right\|_{W_{2}^{-1 / 2}\left(\Gamma_{k}\right)}$ and fix any integer $N \geq 1$. Then

$$
\sum_{k=1}^{N} \alpha_{k}^{2} \leq 2 \sum_{k=1}^{N} \alpha_{k}\left\langle\mu_{k}(f-\bar{f}), v_{k}\right\rangle=2\left\langle f-\bar{f}, \sum_{k=1}^{N} \alpha_{k} \mu_{k}\left(v_{k}-\dot{o}_{k}\right)\right\rangle
$$

where $\dot{v}_{k}$ is the mean value of $v_{k}$ on the surface $\Gamma_{k}$. Clearly, $\mu_{k}=\left(1-\mu_{0}\right) \mu_{k}$ for $k \geq 2$. We also have $\mu_{1}=\left(1-\mu_{0}\right) \mu_{1}$ on $\left[z_{2}, z_{1}\right]$ and $\mu_{1}=1-\mu_{0}$ on $\left[z_{1}, z_{0}\right]$. Thus $\mu_{1}$ can be represented by the product $\left(1-\mu_{0}\right) \nu_{1}$ where

$$
\nu_{1}(z)= \begin{cases}\mu_{1}(z), & z \in\left[z_{2}, z_{1}\right] \\ \lambda_{1}(z), & z \in\left[z_{1}, z_{0}\right] .\end{cases}
$$


We recall that $\lambda_{k} \in C_{0}^{\infty}\left(\Delta_{k}\right)$ is a set of functions for which $\mu_{k} \lambda_{k}=\mu_{k}, k=$ $1,2, \ldots$ Hence $\nu_{1} \in C_{0}^{\infty}\left(z_{2}, z_{0}\right), 0 \leq \nu_{1} \leq 1$. Letting $\nu_{k}=\mu_{k}$ for $k=2,3, \ldots$, we rewrite inequality $(3.9)$ as

$$
\sum_{k=1}^{N} \alpha_{k}^{2} \leq 2\left\langle\left(1-\mu_{0}\right)(f-\bar{f}), \sum_{k=1}^{N} \alpha_{k} \nu_{k}\left(v_{k}-\stackrel{\circ}{v}_{k}\right)\right\rangle .
$$

Assuming that $\nu_{k}(z) v_{k}(x)=0$ outside $\Gamma_{k}$, define a function $v$ on $\Gamma$ by

$$
v(x)=\sum_{k=1}^{N} \alpha_{k} \nu_{k}(z) w_{k}(x), \quad w_{k}(x)=v_{k}(x)-\stackrel{\circ}{v}_{k} .
$$

Let us now estimate $\|v\|_{T(\Omega)}$ from above. Since $\operatorname{supp} v \subset\left\{x \in \Gamma: z \leq z_{0}\right\}$, it suffices to estimate $|v|_{\Gamma}$. Let $x, \xi \in \Gamma$. Clearly,

$$
v(x)-v(\xi)=\sum_{k=1}^{N} \alpha_{k}\left(\nu_{k}(z) w_{k}(x)-\nu_{k}(\zeta) w_{k}(\xi)\right),
$$

and the last sum contains at most four nonzero summands. Therefore

$$
|v(x)-v(\xi)|^{2} \leq 4 \sum_{k=1}^{N} \alpha_{k}^{2}\left(\nu_{k}(z) w_{k}(x)-\nu_{k}(\zeta) w_{k}(\xi)\right)^{2}
$$

hence

$$
|v|_{\Gamma}^{2} \leq 4 \sum_{k=1}^{N} \alpha_{k}^{2}\left|\nu_{k} w_{k}\right|_{\Gamma}^{2}
$$

Note that the function

$$
\Gamma \times \Gamma \ni(x, \xi) \mapsto \nu_{k}(z) w_{k}(x)-\nu_{k}(\zeta) w_{k}(\xi)
$$

is zero if $x \notin \Gamma_{k}$ and $\xi \notin \Gamma_{k}$ so that

$$
\left|\nu_{k} w_{k}\right|_{\Gamma}^{2} \leq\left[\nu_{k} w_{k}\right]_{\Gamma_{k}}^{2}+2 \int_{\Gamma_{k}}\left|\nu_{k}(z) w_{k}(x)\right|^{2} d s_{x} \int_{\left\{\xi \notin \Gamma_{k}:|z-\zeta|<M(z, \zeta)\right\}}|x-\xi|^{-n} d s_{\xi},
$$

where $[\cdot]_{\Gamma_{k}}$ is the seminorm defined in (1.1). Next, for $z \in \operatorname{supp} \nu_{k}, \zeta \notin \Delta_{k}(2.4)$ implies that $|z-\zeta| \geq c \varphi\left(z_{k}\right)$, hence we have $|x-\xi| \sim \varphi\left(z_{k}\right)$ in the last integral. Thus the second term on the right-hand side of (3.12) does not exceed

$$
c \varphi\left(z_{k}\right)^{-1} \int_{\Gamma_{k}}\left|v_{k}(x)-\stackrel{\circ}{v}_{k}\right|^{2} d s_{x}
$$

with a constant independent of $k$ and $v_{k}$. Finally, by Lemma 3 expression (3.13) is not greater than $c\left[v_{k}\right]_{\Gamma_{k}}^{2}$.

To estimate $\left[\nu_{k} w_{k}\right]_{\Gamma_{k}}$, we first use the inequality

$$
\left[\nu_{k} w_{k}\right]_{\Gamma_{k}}^{2} \leq c\left[v_{k}\right]_{\Gamma_{k}}^{2}+c \int_{\Gamma_{k}}\left|v_{k}(x)-\stackrel{\circ}{v}_{k}\right|^{2} d s_{x} \int_{\Gamma_{k}} \frac{\left|\nu_{k}(z)-\nu_{k}(\zeta)\right|^{2}}{|x-\xi|^{n}} d s_{\xi}
$$


According to (2.4), one has

$$
\left|\nu_{k}(z)-\nu_{k}(\zeta)\right| \leq c \varphi\left(z_{k}\right)^{-1}|z-\zeta|
$$

therefore the last integral over $\Gamma_{k}$ is dominated by

$$
c \varphi\left(z_{k}\right)^{-2} \int_{\Gamma_{k}}|x-\xi|^{2-n} d s_{\xi} \leq c \varphi\left(z_{k}\right)^{-1} .
$$

Then, by Lemma 3 the second term on the right-hand side of (3.14) is majorized by $c\left[v_{k}\right]_{\Gamma_{k}}^{2}$.

Thus we have established that

$$
\left|\nu_{k} w_{k}\right|_{\Gamma} \leq c\left[v_{k}\right]_{\Gamma_{k}}
$$

with a constant independent of $k$ and $v_{k}$. Since $\left\|v_{k}\right\|_{W_{2}^{1 / 2}\left(\Gamma_{k}\right)} \leq 1$, it follows from the last estimate and (3.11) that

$$
|v|_{\Gamma}^{2} \leq c \sum_{k=1}^{N} \alpha_{k}^{2}
$$

So the expression on the right-hand side of (3.10) does not exceed

$$
c\left\|\left(1-\mu_{0}\right)(f-\bar{f})\right\|_{T(\Omega) *}\left(\sum_{k=1}^{N} \alpha_{k}^{2}\right)^{1 / 2} .
$$

Now (3.10) gives

$$
\sum_{k=1}^{N} \alpha_{k}^{2} \leq c\left\|\left(1-\mu_{0}\right)(f-\bar{f})\right\|_{T(\Omega)^{*}}\left(\sum_{k=1}^{N} \alpha_{k}^{2}\right)^{1 / 2} .
$$

with a constant independent of $\alpha_{k}=\left\|\mu_{k}(f-\bar{f})\right\|_{W_{2}^{-1 / 2}\left(\Gamma_{k}\right)}$ and $N$. Hence we obtain (3.2).

(ii) Let $v \in T(\Omega)$. Then

$$
\begin{aligned}
\sum_{k \geq 1}\left|\left\langle\lambda_{k} f_{k}, v\right\rangle\right| & \leq \sum_{k \geq 1}\left|\left\langle\lambda_{k} f_{k}, \sum_{|k-i| \leq 1} \mu_{i}\left(v-\stackrel{\circ}{v}_{k}\right)\right\rangle\right| \\
& \leq \sum_{k \geq 1} \sum_{|k-i| \leq 1}\left\|\lambda_{k} f_{k}\right\|_{W_{2}^{-1 / 2}\left(\Gamma_{k}\right)}\left\|\mu_{i}\left(v-\stackrel{\circ}{v}_{k}\right)\right\|_{W_{2}^{1 / 2}\left(\Gamma_{k}\right)},
\end{aligned}
$$

where $\stackrel{\circ}{v}_{k}$ is the mean value of $v$ on $\Gamma_{k}$. Clearly

$$
\left\|\mu_{i}\left(v-\stackrel{\circ}{v}_{k}\right)\right\|_{W_{2}^{1 / 2}\left(\Gamma_{k}\right)} \leq\left\|v-\stackrel{\circ}{v}_{k}\right\|_{L_{2}\left(\Gamma_{k}\right)}+\left[\mu_{i}\left(v-\stackrel{\circ}{v}_{k}\right)\right]_{\Gamma_{k}} .
$$

We estimate the first term on the right by Lemma 3 :

$$
\left\|v-\stackrel{\circ}{v}_{k}\right\|_{L_{2}\left(\Gamma_{k}\right)} \leq c \varphi\left(z_{k}\right)^{1 / 2}[v]_{\Gamma_{k}} .
$$

Next, we have

$$
\left[\mu_{i}\left(v-\stackrel{\circ}{v}_{k}\right)\right]_{\Gamma_{k}}^{2} \leq c[v]_{\Gamma_{k}}^{2}+c \int_{\Gamma_{k}}\left|v(x)-\stackrel{\circ}{v}_{k}\right|^{2} d s_{x} \int_{\Gamma_{k}} \frac{\left|\mu_{i}(z)-\mu_{i}(\zeta)\right|^{2}}{|x-\xi|^{n}} d s_{\xi}
$$


Since $|k-i| \leq 1$, it follows that $\left|\mu_{i}(z)-\mu_{i}(\zeta)\right| \leq c \varphi\left(z_{k}\right)^{-1}|z-\zeta|$, and the second term on the right-hand side of (3.16) is dominated by the first term in the same way as the second term on the right in (3.14) has been majorized by the first one. Thus we have shown that

$$
\left\|\mu_{i}\left(v-\stackrel{\circ}{v}_{k}\right)\right\|_{W_{2}^{1 / 2}\left(\Gamma_{k}\right)} \leq c[v]_{\Gamma_{k}}
$$

Now (3.15) implies the estimate

$$
\begin{aligned}
& \sum_{k \geq 1}\left|\left\langle\lambda_{k} f_{k}, v\right\rangle\right| \leq c \sum_{k \geq 1}\left\|\lambda_{k} f_{k}\right\|_{W_{2}^{-1 / 2}\left(\Gamma_{k}\right)}[v]_{\Gamma_{k}} \\
& \leq c\left(\sum_{k \geq 1}\left\|\lambda_{k} f_{k}\right\|_{W_{2}^{-1 / 2}\left(\Gamma_{k}\right)}^{2}\right)^{1 / 2}\left(\sum_{k \geq 1}[v]_{\Gamma_{k}}^{2}\right)^{1 / 2} .
\end{aligned}
$$

We observe that $|z-\zeta|<2 M(z, \zeta)$ for $x, \xi \in \Gamma_{k}$, whence

$$
\sum_{k \geq 1}[v]_{\Gamma_{k}}^{2} \leq \int_{\{x, \xi \in \Gamma:|\zeta-z|<2 M(z, \zeta)\}}|v(x)-v(\xi)|^{2} \frac{d s_{x} d s_{\xi}}{|x-\xi|^{n}}
$$

In view of Lemma 2 the right-hand side of the last inequality does not exceed

$$
c\left|\left(1-\mu_{0}\right) v\right|_{\Gamma}^{2}+c\left\|\mu_{0} v\right\|_{W_{2}^{1 / 2}\left(\Gamma_{0}\right)}^{2}
$$

which is not greater than $c\|v\|_{T(\Omega)}^{2}$ by Remark 1. Now (3.17) implies that definition (3.4) is correct, $f^{(3)}$ is continuous and (3.5) holds.

The inclusion $f^{(1)} \in W_{2}^{-1 / 2}\left(\Gamma_{0}\right)$ follows from Gagliardo's theorem and Remark 1. Since $T(\Omega) \subset W_{2}^{1 / 2}\left(\Gamma_{0}\right)$, we also have $f^{(1)} \in T(\Omega)^{*}$.

The proof of the continuity, in $T(\Omega)$, of the functional $f^{(2)}$ given by (3.6) for $g \in \bar{W}_{2}^{1 / 2}(0,1)^{*}$ is carried out in the same way as in assertion (i). The proof of the theorem is finished.

We now state some consequences of the theorem.

Corollary 1. Let $\Omega$ be a domain with an outer peak. If $f$ is a linear functional defined on $T(\Omega)$ and $f$ can be represented as the sum of three terms $f^{(1)}, f^{(2)}$, $f^{(3)}$ with properties described in assertion (ii) of the theorem, then the Neumann problem (1.2), (1.3) has a unique solution.

Corollary 2. Let $\Omega$ be a domain with outer peak and let $f$ be a linear functional defined on $T(\Omega)$. For $v \in T(\Omega)$, supp $v \subset \Gamma$, define

$$
\langle\bar{f}, v\rangle=\langle f, \bar{v}\rangle \text {. }
$$

Then the functional $\left(1-\mu_{0}\right)(f-\bar{f})$ is continuous on $T(\Omega)$ if and only if

$$
\sum_{k \geq 1}\left\|\mu_{k}(f-\bar{f})\right\|_{W_{2}^{-1 / 2}\left(\Gamma_{k}\right)}^{2}<\infty
$$


Furthermore, the following relation holds

$$
\left(\sum_{k \geq 1}\left\|\mu_{k}(f-\bar{f})\right\|_{W_{2}^{-1 / 2}\left(\Gamma_{k}\right)}^{2}\right)^{1 / 2} \sim\left\|\left(1-\mu_{0}\right)(f-\bar{f})\right\|_{T(\Omega)^{*}}
$$

with constants depending only on $\Omega$.

Proof. The lower bound for $\left\|\left(1-\mu_{0}\right)(f-\bar{f})\right\|_{T(\Omega) *}$ was obtained in assertion (i) of the theorem. To establish the upper bound, consider the functionals $f_{k}=\mu_{k}(f-\bar{f})$ satisfying (3.18) for $k=1,2, \ldots$ It is clear that $\operatorname{supp} f_{k} \subset \Gamma_{k}$ and that $\left\langle f_{k}, v\right\rangle=0$ if $v \in W_{2}^{1 / 2}\left(\Gamma_{k}\right), v(x)$ depends only on $z$. We have $\lambda_{k} f_{k}=$ $\mu_{k}(f-\bar{f})=f_{k}$ for each $k \geq 1$, and assertion (ii) of the theorem gives

$$
\left\|\sum_{k \geq 1} f_{k}\right\|_{T(\Omega)^{*}} \leq c\left(\sum_{k \geq 1}\left\|f_{k}\right\|_{W_{2}^{-1 / 2}\left(\Gamma_{k}\right)}^{2}\right)^{1 / 2}
$$

thus concluding the proof.

The preceding assertion enables us to prove the continuity of the linear map

$$
T(\Omega) \ni v \mapsto v-\bar{v} \in L_{q}(\Gamma)
$$

with a maximal Sobolev exponent $q$ and to establish the inclusion $f-\bar{f} \in T(\Omega)^{*}$ for $f \in L_{q^{\prime}}(\partial \Omega)$ with a minimal possible exponent $q^{\prime}=q /(q-1)$.

Corollary 3. Let $\Omega$ be a domain with an outer peak, let $q=2(n-1) /(n-2)$ and $q^{\prime}=q /(q-1)$. If $f \in L_{q^{\prime}}(\partial \Omega)$, then the functional

$$
T(\Omega) \ni v \mapsto\left\langle\left(1-\mu_{0}\right)(f-\bar{f}), v\right\rangle=\int_{\Gamma} f(x)(v(x)-\bar{v}(z))\left(1-\mu_{0}(z)\right) d s_{x}
$$

is in $T(\Omega)^{*}$ and the estimate

$$
\left\|\left(1-\mu_{0}\right)(f-\bar{f})\right\|_{T(\Omega)^{*}} \leq c\|f-\bar{f}\|_{L_{q^{\prime}}(\Gamma)}
$$

is valid with a constant independent of $f$. Furthermore, for all $v \in T(\Omega)$ we have

$$
\|v-\bar{v}\|_{L_{q}(\Gamma)} \leq c\|v\|_{T(\Omega)}
$$

with a constant independent of $v$.

Proof. According to Corollary 2 we should bound the sum on the left of (3.18). Let $v \in W_{2}^{1 / 2}\left(\Gamma_{k}\right)$. An application of Hölder's inequality gives

$$
\begin{aligned}
\left|\left\langle\mu_{k}(f-\bar{f}), v\right\rangle\right| & =\left|\int_{\Gamma_{k}} \mu_{k}(f-\bar{f})\left(v-\stackrel{\circ}{v}_{k}\right) d s_{x}\right| \\
& \leq\|f-\bar{f}\|_{L_{q^{\prime}}\left(\Gamma_{k}\right)} \| \mu_{k}\left(v-\stackrel{\circ}{v}_{k} \|_{L_{q}\left(\Gamma_{k}\right)},\right.
\end{aligned}
$$

where, as above, $\stackrel{\circ}{v}_{k}$ denotes the mean value of $v$ on $\Gamma_{k}$. Since

$$
\Omega_{k}=\left\{(y, z): z \in\left(z_{k+1}, z_{k-1}\right), y / \varphi(z) \in \omega\right\}
$$


is a domain of the class $C^{0,1}$, Sobolev's theorem applies. Hence for any $u \in$ $W_{2}^{1 / 2}\left(\partial \Omega_{k}\right)$ the following estimate holds:

$$
\begin{gathered}
\|u\|_{L_{q}\left(\partial \Omega_{k}\right)} \leq c \varphi\left(z_{k}\right)^{\frac{n-1}{q}-\frac{n-1}{2}}\|u\|_{L_{2}\left(\partial \Omega_{k}\right)}+c \varphi\left(z_{k}\right)^{1+\frac{n-1}{q}-\frac{n}{2}}[u]_{\partial \Omega_{k}} \\
=c \varphi\left(z_{k}\right)^{-1 / 2}\|u\|_{L_{2}\left(\partial \Omega_{k}\right)}+c[u]_{\partial \Omega_{k}} .
\end{gathered}
$$

Here $[\cdot]_{\partial \Omega_{k}}$ is the seminorm defined in (1.1). Inserting $u=\mu_{k}\left(v-\dot{\circ}_{k}\right)$ into the last inequality (we assume that $u=0$ on $\partial \Omega_{k} \backslash \Gamma_{k}$ ) and, using Lemma 3 to bound the first term on the right, we obtain

$$
\| \mu_{k}\left(v-\stackrel{\circ}{v}_{k} \|_{L_{q}\left(\Gamma_{k}\right)} \leq c[v]_{\Gamma_{k}}+c\left[\mu_{k}\left(v-\stackrel{\circ}{v}_{k}\right]_{\partial \Omega_{k}} .\right.\right.
$$

The last term will be estimated as follows. First note that

$$
\begin{aligned}
{\left[\mu_{k}\left(v-\dot{\circ}_{k}\right]_{\partial \Omega_{k}}^{2}=\right.} & {\left[\mu_{k}\left(v-\dot{\circ}_{k}\right]_{\Gamma_{k}}^{2}\right.} \\
& +2 \int_{\Gamma_{k}}\left|\mu_{k}(z)\left(v(x)-\dot{\circ}_{k}\right)\right|^{2} d s_{x} \int_{\partial \Omega_{k} \backslash \Gamma_{k}}|x-\xi|^{-n} d s_{\xi} .
\end{aligned}
$$

If $x=(y, z) \in \Gamma_{k}, z \in \operatorname{supp} \mu_{k}$, and $\xi \notin \Gamma_{k}$, then $|x-\xi| \geq c \varphi\left(z_{k}\right)$ in view of (2.4). Therefore the last term in (3.23) does not exceed

$$
c \varphi\left(z_{k}\right)^{-1} \int_{\Gamma_{k}}\left|v(x)-\stackrel{\circ}{*}_{k}\right|^{2} d s_{x}
$$

which is not greater than $c[v]_{\Gamma_{k}}^{2}$ by Lemma 3 . Then we dominate the quantity $\left[\mu_{k}\left(v-\dot{v}_{k}\right]_{\Gamma_{k}}^{2}\right.$ by the right-hand side of inequality (3.14) where $v_{k}(x)$ should be replaced by $v(x)$ and $\nu_{k}$ should be replaced by $\mu_{k}$. The same argument as in Theorem 1 gives

$$
\left[\mu_{k}\left(v-\dot{\circ}_{k}\right]_{\Gamma_{k}} \leq c[v]_{\Gamma_{k}}\right.
$$

Inequalities (3.22), (3.23) combined with the last estimates show that

$$
\| \mu_{k}\left(v-\stackrel{\circ}{v}_{k} \|_{L_{q}\left(\Gamma_{k}\right)} \leq c[v]_{\Gamma_{k}} .\right.
$$

This and (3.21) imply

$$
\left\|\mu_{k}(f-\bar{f})\right\|_{W_{2}^{-1 / 2}\left(\Gamma_{k}\right)} \leq c\|f-\bar{f}\|_{L_{q^{\prime}}\left(\Gamma_{k}\right)} .
$$

Thus

$$
\left(\sum_{k \geq 1}\left\|\mu_{k}(f-\bar{f})\right\|_{W_{2}^{-1 / 2}\left(\Gamma_{k}\right)}^{2}\right)^{1 / 2} \leq c\left(\sum_{k \geq 1}\|f-\bar{f}\|_{L_{q^{\prime}}\left(\Gamma_{k}\right)}^{2}\right)^{1 / 2} .
$$

Applying an algebraic inequality

$$
\left(\sum_{k \geq 1} a_{k}^{2}\right)^{1 / 2} \leq\left(\sum_{k \geq 1} a_{k}^{q^{\prime}}\right)^{1 / q^{\prime}}, \quad a_{k} \geq 0, \quad 0<q^{\prime}<2,
$$

we majorize the left-hand side of (3.24) by the expression $c\|f-\bar{f}\|_{L_{q^{\prime}}(\Gamma)}$. Now (3.19) follows from Corollary 2.

Turning to (3.20), we note that

$$
\left\langle f,\left(1-\mu_{0}\right)(v-\bar{v})\right\rangle=\left\langle\left(1-\mu_{0}\right)(f-\bar{f}), v\right\rangle
$$


and that $\|\bar{f}\|_{L_{q^{\prime}}(\Gamma)} \leq c\|f\|_{L_{q^{\prime}}(\Gamma)}$. So (3.19) implies

$$
\left|\left\langle f,\left(1-\mu_{0}\right)(v-\bar{v})\right\rangle\right| \leq c\|f\|_{L_{q^{\prime}}(\Gamma)}\|v\|_{T(\Omega)}
$$

for all $f \in L_{q^{\prime}}(\Gamma)$ and $v \in T(\Omega)$. Let $V$ denote the unit ball in $T(\Omega)$. For every $v \in V$ and $f \in L_{q^{\prime}}(\Gamma)$ define

$$
F_{v}(f)=\left\langle f,\left(1-\mu_{0}\right)(v-\bar{v})\right\rangle=\int_{\Gamma} f(x)\left(1-\mu_{0}(z)\right)(v(x)-\bar{v}(z)) d s_{x} .
$$

In view of (3.25), the functionals $L_{q^{\prime}}(\Gamma) \ni v \mapsto F_{v}(f)$ are continuous and pointwise bounded for $v \in V$. Hence their norms are uniformly bounded, which means that $\left\|\left(1-\mu_{0}\right)(v-\bar{v})\right\|_{L_{q}(\Gamma)} \leq$ const for $v \in V$. Thus

$$
\left\|\left(1-\mu_{0}\right)(v-\bar{v})\right\|_{L_{q}(\Gamma)} \leq \mathrm{const} \cdot\|v\|_{T(\Omega)}
$$

for all $v \in T(\Omega)$. To conclude the proof of (3.20), we show that

$$
\left\|\mu_{0}(v-\bar{v})\right\|_{L_{q}(\Gamma)} \leq \mathrm{const} \cdot\|v\|_{T(\Omega)} .
$$

By definition (2.2) and Hölder's inequality we obtain

$$
|\bar{v}(z)|^{q} \varphi(z)^{n-2}|\gamma| \leq \int_{y \in \varphi(z) \gamma}|v(y, z)|^{q} d \gamma(y) .
$$

Integration over $z \in\left(z_{1}, 1\right)$ gives

$$
\left\|\mu_{0} \bar{v}\right\|_{L_{q}\left(\Gamma \cap \Gamma_{0}\right)}^{q} \leq c\left\|\mu_{0} v\right\|_{L_{q}\left(\Gamma \cap \Gamma_{0}\right)}^{q} .
$$

It remains to observe that by Sobolev's theorem the last norm does not exceed $c\left\|\mu_{0} v\right\|_{W_{2}^{1 / 2}\left(\Gamma_{0}\right)}$ which, according to Remark 1, is not greater than $\|v\|_{T(\Omega)}$. The result follows.

A combination of Theorem 1 with Corollary 3 enables us to state the following proposition.

Proposition. Let $\Omega$ be a domain with an outer peak and $1 \leq q \leq 2(n-$ $1) /(n-2), q^{-1}+q^{-1}=1$. The following assertions are equivalent.

(A) The Neumann problem (1.2), (1.3) is uniquely solvable for all $f \in L_{q^{\prime}}(\partial \Omega)$.

(B) For all $f \in L_{q^{\prime}}(\partial \Omega)$ the functional

$$
T(\Omega) \ni v \mapsto \int_{\partial \Omega} f v d s_{x}
$$

is continuous.

(C) The space $T(\Omega)$ is continuously imbedded into $L_{q}(\partial \Omega)$.

(D) The map $T(\Omega) \ni v \mapsto \bar{v} \in L_{q}(\Gamma)$ is continuous.

(E) $\bar{W}_{2}^{1 / 2}(0,1)$ is continuously imbedded in the weighted $L_{q}$ with the norm

$$
u \mapsto\left(\int_{0}^{1}|u(z)|^{q} \varphi(z)^{n-2} d z\right)^{1 / q}
$$


Proof. $(\mathrm{A}) \rightarrow(\mathrm{B})$. Let $E: T(\Omega) \rightarrow W_{2}^{1}(\Omega)$ be a linear continuous extension operator. For any given $f \in L_{q^{\prime}}(\partial \Omega)$ let $u$ denote the solution of the problem (1.2), (1.3). Since (1.4) holds, we have

$$
\int_{\partial \Omega} f v d s_{x}=[u, E v] \text { for all } v \in T(\Omega)
$$

so that

$$
\left|\int_{\partial \Omega} f v d s_{x}\right| \leq c\|u\|_{W_{2}^{1}(\Omega)}\|E v\|_{W_{2}^{1}(\Omega)} \leq c\|u\|_{W_{2}^{1}(\Omega)}\|v\|_{T(\Omega)},
$$

and the result follows.

$(\mathrm{B}) \rightarrow(\mathrm{C})$. Using the well known fact that bounded functions are dense in $W_{2}^{1}(\Omega)$, one can easily obtain that bounded functions are also dense in $T(\Omega)$. Let

$$
V=\left\{v \in T(\Omega) \cap L_{\infty}(\partial \Omega):\|v\|_{T(\Omega)} \leq 1\right\} .
$$

For every $v \in V$ consider a functional

$$
L_{q^{\prime}}(\partial \Omega) \ni f \mapsto F_{v}(f)=\int_{\partial \Omega} f v d s_{x} .
$$

Clearly, $F_{v}$ is continuous on $L_{q^{\prime}}(\partial \Omega)$ and, by assumption, $\left|F_{v}(f)\right| \leq c(f)\|v\|_{T(\Omega)}$. Thus $\left\{F_{v}\right\}_{v \in V}$ are pointwise bounded. Hence $\left\|F_{v}\right\|=\|v\|_{L_{q}(\partial \Omega)} \leq$ const for all $v \in V$. This means that

$$
\|v\|_{L_{q}(\partial \Omega)} \leq \mathrm{const} \cdot\|v\|_{T(\Omega)}
$$

for all $v \in T(\Omega) \cap L_{\infty}(\partial \Omega)$. Thus $T(\Omega)$ is continuously imbedded into $L_{q}(\partial \Omega)$.

$(\mathrm{C}) \rightarrow(\mathrm{A})$. Let $f \in L_{q^{\prime}}(\partial \Omega)$ and $v \in W_{2}^{1}(\Omega)$. Then by Hölder's inequality

$$
\left|\int_{\partial \Omega} f v d s_{x}\right| \leq\|f\|_{L_{q^{\prime}}(\partial \Omega)}\|v\|_{L_{q}(\partial \Omega)} .
$$

By assumption, the last norm does not exceed $c\|v\|_{T(\Omega)}$ which is not greater than $c\|v\|_{W_{2}^{1}(\Omega)}$. So the functional on the right in (1.4) is continuous with respect to $v \in W_{2}^{1}(\Omega)$. Hence the Neumann problem is solvable.

The equivalence of (C) and (D) follows from Corollary 3.

$(\mathrm{D}) \rightarrow(\mathrm{E})$. Let $u \in \bar{W}_{2}^{1 / 2}(0,1)$. Suppose that $\lambda \in C^{\infty}(0,1)$ and

$$
0 \leq \lambda \leq 1,\left.\quad \lambda\right|_{\left(0, z_{2}\right)}=1,\left.\quad \lambda\right|_{\left(z_{1}, 1\right)}=0 .
$$

We define $v \in T(\Omega)$ by $v(x)=\lambda(z) u(z)$ for $x \in \Gamma$, and $v=0$ on $\partial \Omega \backslash \Gamma$. Then, since $\left.v\right|_{\Gamma_{0}}=0$, and in view of Lemma 1 , the inequality $\|\bar{v}\|_{L_{q}(\Gamma)} \leq c\|v\|_{T(\Omega)}$ transforms to

$$
\left(\int_{0}^{1}|\lambda(z) u(z)|^{q} \varphi(z)^{n-2} d z\right)^{1 / q} \leq c\|\lambda u\|_{W_{2}^{1 / 2}(0,1)} .
$$


The same reasoning as in the proof of inequality (3.1) in Theorem 1 shows that the right-hand side of the last inequality does not exceed $c(\lambda)\|u\|_{\bar{W}_{2}^{1 / 2}(0,1)}$. Thus we have

$$
\|\lambda u\|_{L_{q}(\Gamma)} \leq c\|u\|_{\bar{W}_{2}^{1 / 2}(0,1)}
$$

On the other hand,

$$
\|(1-\lambda) u\|_{L_{q}(\Gamma)} \leq c\left(\int_{z_{2}}^{1}|u(z)|^{q} d z\right)^{1 / q}
$$

Since $W_{2}^{1 / 2}\left(z_{2}, 1\right)$ is continuously imbedded into $L_{q}\left(z_{2}, 1\right)$, it follows that

$$
\left(\int_{0}^{1}|(1-\lambda(z)) u(z)|^{q} \varphi(z)^{n-2} d z\right)^{1 / q} \leq c\|u\|_{W_{2}^{1 / 2}\left(z_{2}, 1\right)} \leq c\|u\|_{\bar{W}_{2}^{1 / 2}(0,1)} .
$$

The last inequality and (3.26) give the desired result.

$(\mathrm{E}) \rightarrow(\mathrm{D})$. Let $v \in T(\Omega)$. Lemma 1 says that $\|\bar{v}\|_{\bar{W}_{2}^{1 / 2}(0,1)} \leq c\|v\|_{T(\Omega)}$. Hence

$$
\left(\int_{0}^{1}|\bar{v}(z)|^{q} \varphi(z)^{n-2} d z\right)^{1 / q} \leq c\|v\|_{T(\Omega)}
$$

which means the continuity of the map $T(\Omega) \ni v \mapsto \bar{v} \in L_{q}(\Gamma)$. The proof of the proposition is finished.

The results of the paper [6] enable us to complete the above proposition by one more assertion. Any of statements (A)-(E) above is equivalent to the following one:

(F) If $1 \leq q<2$, then

$$
\int_{0}^{1}\left[\int_{0}^{z} \varphi(t)^{n-2} d t\left(\int_{z}^{1} \frac{d t}{\varphi(t)^{n-1}}\right)^{q-1}\right]^{\frac{2}{2-q}} \frac{d z}{\varphi(z)^{n-1}}<\infty
$$

and if $q \geq 2$, then

$$
\sup _{r \in(0,1)}\left(\int_{0}^{r} \varphi(z)^{n-2} d z\right)^{1 / q}\left(\int_{r}^{1} \varphi(z)^{1-n} d z\right)^{1 / 2}<\infty .
$$

\section{A Domain with an Inner Peak}

In this section we describe the space dual of $T W_{2}^{1}$ for a domain with the vertex of an inner peak at the boundary. Let $\Omega \subset \mathbf{R}^{n}$ be the domain with an outer peak as above. Here we introduce a domain with an inner peak as $\mathbf{R}^{n} \backslash \bar{\Omega}$. For brevity, we write below $T\left(\mathbf{R}^{n} \backslash \bar{\Omega}\right)$ instead of $T W_{2}^{1}\left(\mathbf{R}^{n} \backslash \bar{\Omega}\right)$. According 
to Theorems 7.3 and 7.4 .2 in [5] (see also [3], [4]), the norm in $T\left(\mathbf{R}^{n} \backslash \bar{\Omega}\right.$ ) is equivalent to the norm

$$
\left(\|v\|_{W_{2}^{1 / 2}\left(\Gamma_{0}\right)}^{2}+\int_{\Gamma} v(x)^{2} \varphi(z)^{-1} d s_{x}+|v|_{\Gamma}^{2}\right)^{1 / 2}
$$

for $n>3$ and to the norm

$$
\begin{gathered}
\left\{\|v\|_{W_{2}^{1 / 2}\left(\Gamma_{0}\right)}^{2}+|v|_{\Gamma}^{2}+\int_{\Gamma} \frac{v(x)^{2} d s_{x}}{\varphi(z) \log (z / \varphi(z)}\right. \\
\left.+\iint_{\{x, \xi \in \Gamma:|z-\zeta|>M(z, \zeta)\}}|v(x)-v(\xi)|^{2} \frac{M(z, \zeta)^{-2} \chi_{(1 / 2,2)}(z / \zeta) d s_{x} d s_{\xi}}{|x-\xi|(\log (1+|x-\xi| / M(z, \zeta)))^{2}}\right\}^{1 / 2}
\end{gathered}
$$

for $n=3$. Here we have used the same notation as in (2.6), and if $n=3$ it must be additionally assumed that $\left.\varphi^{\prime}(z)=O(\varphi(z) / z)\right)$ for $z \rightarrow 0$.

The norms just indicated induce the following norms in $\bar{W}_{2}^{1 / 2}(0,1)$, which are the restriction of norms in $T\left(\mathbf{R}^{n} \backslash \bar{\Omega}\right)$ to the subspace of functions with support in $\Gamma$, depending only on $z$. Thus for $n>3$ we can take the norm

$$
\left(\int_{0}^{1} u(z)^{2} \varphi(z)^{n-3} d z+\iint_{\{z, \zeta \in(0,1):|\zeta-z|<M(z, \zeta)\}}|u(z)-u(\zeta)|^{2} \frac{M(z, \zeta)^{n-2}}{|z-\zeta|^{2}} d z d \zeta\right)^{\frac{1}{2}}
$$

and for $n=3$ the norm in $\bar{W}_{2}^{1 / 2}(0,1)$ can be written in the form

$$
\left(\int_{0}^{1} \frac{u(z)^{2} d z}{\log (z / \varphi(z))}+\iint_{\left\{z, \zeta \in(0,1), z^{-1} \zeta \in(1 / 2,2)\right\}} \frac{|u(z)-u(\zeta)|^{2}}{|z-\zeta|} Q\left(\frac{|z-\zeta|}{M(z, \zeta)}\right) d z d \zeta\right)^{\frac{1}{2}}
$$

where

$$
Q(t)=\left\{\begin{array}{l}
t^{-1}, \quad t \in(0,1) \\
(\log (e t))^{-1}, t>1
\end{array}\right.
$$

The following theorem gives a description of the space $\left(T W_{2}^{1}\right)^{*}$ for a domain with an inner peak.

Theorem 2. Let $\Omega$ be a domain with an outer peak and let $\left\{\mu_{k}\right\}_{k \geq 0}$ be the partition of unity from Theorem 1 .

(i) Any functional $f$ in $T\left(\mathbf{R}^{n} \backslash \bar{\Omega}\right)^{*}$ can be represented as the sum

$$
f=\mu_{0} f+\left(1-\mu_{0}\right) \bar{f}+\left(1-\mu_{0}\right)(f-\bar{f}),
$$

where each term belongs to the same space. Furthermore the first term is in $W_{2}^{-1 / 2}\left(\Gamma_{0}\right)$ and has support in $\Gamma_{0}$. The second term is supported in $\{x \in \Gamma: z \leq$ $\left.z_{0}\right\}$ and belongs to the space $\bar{W}_{2}^{1 / 2}(0,1)^{*}$ in the sense that estimate (3.1) holds, where $\Omega$ on the right-hand side should be replaced by $\mathbf{R}^{n} \backslash \bar{\Omega}$. For the third term we have

$$
\left(1-\mu_{0}\right)(f-\bar{f})=\sum_{k \geq 1} \mu_{k}(f-\bar{f})
$$


and (3.2) is valid if $\Omega$ is replaced by $\mathbf{R}^{n} \backslash \bar{\Omega}$ on the right.

(ii) Suppose that $f_{k} \in W_{2}^{-1 / 2}\left(\Gamma_{k}\right)$ satisfy condition (ii) of Theorem $1, k=$ $1,2, \ldots$ Suppose further that $g$ and $h$ belong to $\bar{W}_{2}^{1 / 2}(0,1)^{*}$ and $W_{2}^{-1 / 2}\left(\Gamma_{0}\right)$, respectively. Then each functional $f^{(1)}=\mu_{0} h$,

$$
\begin{gathered}
T\left(\mathbf{R}^{n} \backslash \bar{\Omega}\right) \ni v \mapsto\left\langle f^{(2)}, v\right\rangle=\left\langle g,\left(1-\mu_{0}\right) \bar{v}\right\rangle, \\
T\left(\mathbf{R}^{n} \backslash \bar{\Omega}\right) \ni v \mapsto\left\langle f^{(3)}, v\right\rangle=\sum_{k \geq 1}\left\langle\lambda_{k} f_{k}, v\right\rangle
\end{gathered}
$$

is continuous in $T\left(\mathbf{R}^{n} \backslash \bar{\Omega}\right)$. Moreover, $f^{(1)} \in W_{2}^{-1 / 2}\left(\Gamma_{0}\right)$, and the norm of $f^{(3)}$ is dominated by the right-hand side of (3.5).

The proof of Theorem 2 is similar to that of Theorem 1.

\section{REFERENCES}

1. E. Gagliardo, Caratterizzazioni delle tracce sulla frontiera relative ad alcune classi di funzioni in $n$ variabili. (Italian) Rend. Sem. Mat. Univ. Padova 27(1957), 284-305.

2. V. P. Glushko, On domains which are star-like relative to a sphere. (Russian) Dokl. Akad. Nauk SSSR 144(1962), 1215-1216; English transl.: Soviet Math. 3(1962), 878-879.

3. V. G. MAZ'YA, Functions with a finite Dirichlet integral in a domain with a cusp at the boundary. (Russian) Investigations on linear operators and the theory of functions, XII. Zap. Nauchn. Sem. Leningrad. Otdel. Mat. Inst. Steklov. (LOMI) 126(1983), 117-137.

4. V. G. MaZ'ya and S. V. PoborChi, Traces of functions in Sobolev spaces on a boundary of a domain with a cusp. (Russian) Trudy Inst. Mat. (Novosibirsk) 14(1989), Sovrem. Probl. Geom. Analiz., 182-208; English transl.: Siberian Adv. Math. 1(1991), No. 3, $75-107$.

5. V. G. MaZ'ya and S. V. Poborchi, Differentiable functions on bad domains. World Scientific Publishing Co., Inc., River Edge, NJ, 1997.

6. S. V. Poborchi, Continuity of the boundary trace operator $W_{p}^{1}(\Omega) \rightarrow L_{Q}(\partial \Omega)$ for a domain with outward peak. Vestnik St. Petersburg Univ. Math. 38(2005), No. 3, 37-44 (2006).

7. S. L. Sobolev, Some applications of functional analysis in mathematical physics. (Russian) Izdat. Leningrad. Gos. Univ., Leningrad, 1950; Third edition: Nauka, Moscow, 1988. English transl.: Translations of Mathematical Monographs, 90. American Mathematical Society, Providence, RI, 1991.

8. E. M. Stein, Singular integrals and differentiability properties of functions. Princeton Mathematical Series, No. 30. Princeton University Press, Princeton, N.J. 1970.

(Received 17.11.2006)

Authors' addresses:

V. G. Maz'ya

Department of Mathematics

Linköping University

58183 Linköping, Sweden

E-mail: vlmaz@mai.liu.se
S. V. Poborchi

St. Petersburg State University

Department of Mathematics and Mechanics

St. Petersburg, Peterhoff

Bibliotechnaya pl. 2, 198904 Russia

E-mail: Sergei.Poborchi@paloma.spbu.ru 